\title{
Prediction of genetic mutations of equine influenza virus related to adaptive determination nuclear export ribonucleoprotein complex
}

\author{
Mohamed Boukharta ${ }^{1}$, Yassine Kasmi ${ }^{2}$, Fathiah Zakham ${ }^{2}$, Hamid El Amri ${ }^{1}$ and My Mustapha Ennaji $i^{*}$
}

\begin{abstract}
Background: Segment eight (08) of equine influenza virus strain (H3N8) encodes for two non-structural proteins: NS1 (non-structural protein number 1) and NS2, also called NEP (nuclear export protein), via alternative splicing of the pre-messenger RNA transcripts. The NS1 inhibits the cell defense system, while the NEP activates the translocation of the ribonucleoprotein complex to the cytoplasm, a key step in viral assembly. Mutations in this protein could influence viral multiplication.
\end{abstract}

Results: In this present study, we used computational and in silico tools to investigate the impact of mutations on the three-dimensional structure of the CRM1 of NEP proteins of three equine influenza strains ( $\mathrm{H} 3 \mathrm{~N} 8$ ) isolated in Morocco (A/equine/Nador/1/1997 (H3N8), A/equine/Essaouira/2/2004 (H3N8), and A/equine/Essaouira/3/2004 (H3N8)). Compared to the reference strain, the two strains of Essaouira showed no mutations in the domain-binding CRM1 of the NEP protein, while the A/equine/Nador/1/1997 strain (H3N8) had two mutations at the T/33/I and $\mathrm{Q} / 34 / \mathrm{R}$ residues. These two mutations corresponded to adaptation phenomenon of the equine cellular environment, since they enhance the interaction between the protein NEP and CRM1 proteins with a greater affinity compared to the reference strain.

Conclusion: The three-dimensional modification of the NEP protein of the A/equine/Nador/1/1997 strain further facilitates nucleocytoplasmic trafficking in equine species.

Keywords: Equine influenza virus (H3N8), Chromosome maintenance protein 1 (CRM1), Influenza virus NEP (NS2), Ribonucleoprotein export

\section{Background}

Equine influenza is an infectious and contagious disease of the upper respiratory tract in horses (Myers \& Wilson, 2006). The causal agent belongs to the Orthomyxoviridae, genus influenza virus type A. Two distinct subtypes of equine influenza viruses were identified: prototype $\mathrm{A} /$ equine/Prague/1/56 (H7N7) and A/equine/Miami/1/63 (H3N8) (Sovinova, Tumova, Pouska, \& Nemec, 1958; Waddell, Teigland, \& Sigel, 1963). The ancestor of the A/

\footnotetext{
* Correspondence: m.ennaji@yahoo.fr; my.ennaji@univh2m.ac.ma 2Laboratory of Virology, Microbiology, Quality and Biotechnologies/ Ecotoxicology and Biodiversity - Team of Virology, Oncology and Medical Biotechnologies, Faculty of Sciences and Techniques, University Hassan II of Casablanca, P O BOX. 146, 20650 Mohammedia, Morocco

Full list of author information is available at the end of the article
}

equine/Miami/1/63 (H3N8) strain is avian, which might have given rise to several viruses antigenically related to animal species, like a virus isolated from horses in Miami in 1963, a virus isolated from ducks in the same year in Ukraine, and another virus linked to this viral family, which might have recombined 5 years later with the Asian strain H2N2, to give rise to the Hong Kong strain (H3N2) (Dea, Elazhary, \& Roy, 1980; Webster, 1998).

The genome of equine influenza virus (H3N8) is composed of eight segments of negative-sense RNA (of approximately $13.6 \mathrm{~Kb}$ ). The segments are encoding for ten proteins. Of which, six segments encode for one protein (hemagglutinin (HA), neuraminidase (NA), nucleoprotein (NP), and three polymerases: PA, PB1, and PB2), 
the seventh segment is coding for two proteins of the matrix (M1 and M2), and the last one encodes for two non-structural proteins (NS1) and (NS2/NEP (nuclear export protein)) (Brown, 2000; Watanabe et al., 2001)

The NP oligomers around the RNA confers helical symmetry on each of the eight segments. The structural RNA forms three polymerases (PA, PB1, and PB2). The NP forms the viral ribonucleoprotein complex for each segment (vRNP, $\sim 250 \mathrm{kD}$ ), which is essential for the transcription and replication of viral genome (Klaus, Ruigrok, \& Baudin, 1997; Mena et al., 1999; Voeten et al., 2000)

The infection cycle begins with the attachment of the viral particle to the sialic acids of the cell membrane (Temoltzin-Palacios \& Thomas, 1994). Endosomal acidification causes a three-dimensional modification of HA, which is crucial for the fusion of the viral and the endosomal membranes (Chen, Skehel, \& Wiley, 1999; Skehel \& Waterfield, 1975; Smirnova et al., 2009). Activation of the proton channels of the virion (M2) results in the dissociation of the vRNP complexes from the M1 matrix proteins that provide the stability to the viral architecture via the interaction with the envelope and the viral nucleocapsid (Castrucci \& Kawaoka, 1995; Elton, Medcalf, Bishop, Harrison, \& Digard, 1999; Ito et al., 1999).

The vRNPs that were released into the cytoplasm would be translocated to the nucleus to be transcribed and replicated. For this purpose, the viral genome uses the $\alpha / \beta$ import-specific nuclear import system that requires the presence of a nuclear localization signal (NLS) (Iwatsuki-Horimoto et al., 2001).

Nuclear export of vRNPs is a crucial step for the viral cycle (Iwatsuki-Horimoto et al., 2001) and is governed by the newly synthesized viral proteins NP, M1, and NEP which are introduced to the nucleus via the nuclear import system (Neumann, Hughes, \& Kawaoka, 2000).

The nuclear export protein (NEP) $(14 \mathrm{kDa}, 121 \mathrm{aa})$ binds directly to the CRM1 cell export due to its viral export motif nuclear export signal (NES) (11-23 aa) and then by its N-terminal domain of binding to CRM1 (158 aa) (Darapaneni, Prabhaker, \& Kukol, 2009). The NEP also binds to the M1 protein via its C-terminal domain M1 ranging from residue 59 to 116 aa. Various studies showed that NEP, through the use of specific signal NES, is assimilated to an adapter between vRNPs and the nuclear export system. The NES are consensus sequences rich in hydrophobic amino acids, particularly leucines. The N-terminal domain of NEP mediates the RanGTP-dependent binding to CRM1 (Ran (RAs-related nuclear protein)) (Akarsu et al., 2003; Neumann et al., 2000), and the whole is translocated into the cytoplasm via the nuclear pore complex (NPC) nucleopines (Nups) (Fornerod, Ohno, Yoshida, \& Mattaj, 1997). Hydrolysis of RanGTP to RanGDP allowed the dissociation of the export complex and release of the viral vRNP cargo into the cytoplasm (Akarsu et al., 2003).

Viral replication in permissive cells is an efficient and rapid process (less than $10 \mathrm{~h}$ ), and globally, after only 6 hours, the first influenza viruses are detached from the infected respiratory epithelial cells (Neumann, Brownlee, Fodor, \& Kawaoka, 2004; Rott, Orlich, \& Scholtissek, 1979).

In this study, we aimed to determine the amino acid sequence of the CRM1 of the NEP of three equine influenza strains isolated in Morocco: A/equine/Nador/1/ 1997 (H3N8), A/equine/Essaouira/2/2004 (H3N8), and A/equine/Essaouira/3/2004 (H3N8), and the impact of the mutations on the cargo binding to the CRM1 and RanGTP export nuclear complex using computational and modeling tools.

\section{Material and methods \\ Viruses}

A/equine/Nador/1/97 (H3N8) was isolated in Nador from a mule after four passages on 11-day-old embryonated specific pathogen-free chicken eggs (ECE4) as described by Kissi, Daoudi, El kantour, Id sisi yahia, and Benazzou (1998). Both A/equine/Essaouira/2/2004 (H3N8) and A/ equine/Essaouira/3/2004 (H3N8) were isolated respectively from an infected donkey and a horse during 2004 outbreaks in Essaouira. The isolates were passaged on Madin-Darby canine kidney cell line after two passages (MDCK2) at $34{ }^{\circ} \mathrm{C}$ in an atmosphere of $5 \% \mathrm{CO}_{2}$ in Eagle's minimum essential media supplemented with $5 \%$ fetal calf serum (Boukharta et al., 2013).

\section{Viral RNA extraction and amplification}

Viral RNA was extracted directly from isolates using a PureLink Viral RNA/DNA Mini-Kit (Invitrogen, Van Allen Way, Carlsbad, CA, USA) following the manufacturer's recommended protocol. Complementary DNA was obtained by RT reactions, which were carried out by using a SuperScript III First-Strand Synthesis System (Life Technologies, Carlsbad, CA, USA).

PCR was performed using a Platinum PCR SuperMix High-Fidelity Kit (Invitrogen, Carlsbad, CA, USA) with cDNA obtained using primers specific for NS1F (ATGG ATTCCAACACTGTGTC) and NS1R (TCAAACTTC $T G(A / G) C T C A A T T G)$ at a final concentration of $0.5 \mu \mathrm{M}$ for primers. Primer design is previously described by Tissier (2008) and was synthesized by the Unité d'Appui Technique à la Recherche Scientifique, Centre National de Recherche Scientifique et Technique (CNRST), Rabat, Morocco. The assay was performed on the SmartCycler instrument (Cepheid, Sunnyvale, CA, USA), using the following polymerase chain reaction protocol: incubation at $95^{\circ} \mathrm{C}$ for $2 \mathrm{~min}$, then 35 cycles of denaturation at $95^{\circ} \mathrm{C}$ for $30 \mathrm{~s}, 52{ }^{\circ} \mathrm{C}$ for $1 \mathrm{~min}$ for hybridization, $72{ }^{\circ} \mathrm{C}$ 
for $30 \mathrm{~s}$, and a final extension for $3 \mathrm{~min}$ at $72{ }^{\circ} \mathrm{C}$ (Boukharta, Azlmat, Elharrak, \& Ennaji, 2015).

\section{Sequencing NS genes}

The amplified PCR NS products were sequenced. Briefly, the PCR products were purified using EXOSAP-IT (USB Corporation, Cleveland, OH, USA) and bi-directionally sequenced by using BigDye1 Terminator v3.1 (Applied Biosystems, Foster City, CA, USA) on a $3130 \times 1$ model sequencer (Applied Biosystems). Analysis of the electrophoregram was carried out with the sequencing analysis software version 5.3.1 (Applied Biosystems) (Boukharta et al., 2015).

\section{CRM1 sequence}

In order to study the interactions between three-dimensional (3D) structures of the chromosome maintenance protein 1 (CRM1) domain of NEP proteins of A/equine/ Nador/1/1997 (H3N8), A/equine/Essaouira/2/2004 (H3N8), and A/equine/Essaouira/3/2004(H3N8) strains and CRM1 of equids, we used the sequence CRM1 (Equus caballus) of NCBI data (accession number XP_001917538.1) (https://www.ncbi.nlm.nih.gov/protein/ XP_001917538.1).

\section{Determination of amino acid sequences of NS genes}

Multiple alignments of the deduced amino acid sequences were used by using the Basic Local Alignment Search Tool (BLAST) (https://blast.ncbi.nlm.nih.gov/ Blast.cgi). Comparing NEP protein of Moroccan isolates to the NEP reference strain (A/equine/Miami/1/1963) (accession number: ABY81498), the amino acid substitutions were involved in the CRM1 domain of NEP.

\section{Modeling protein structures}

The amino acid sequences of NEP proteins of three Moroccan strains and the CRM1 protein (Equus caballus, XP_001917538.1) were aligned by BLAST (protein-protein) (https://blast.ncbi.nlm.nih.gov/ Blast.cgi), to identify the species and to investigate the existence of crystallized structures at the database of specific crystallized proteins. No structure was crystallized for the equine CRM1 and NEP proteins. Subsequently, the sequences were submitted to the SWISS-MODEL server (Biasini et al., 2014) in order to predict the homology of the 3D structure of the NEP and CRM1 proteins (Equus caballus). Only three-dimensional proteomic structures with an identity percentage greater than $80 \%$ and having a statistically significant homology ( $e$ value 0.001$)$ were chosen. However, the sequences that have not fulfilled all the conditions of validation and filtration in homology were modeled by ab initio computational approach with the I-TASSER server, in order to predict the 3D structure of the protein. The ab initio computational approach allows also to predict the biological function of domains of proteins (Zhang, 2008). The best model obtained by the ab initio approach has undergone model evaluation posttests based on the Ramachandran and Progress server technique (Berjanskii et al., 2010).

\section{Molecular docking}

The effect of mutations on the affinity of the NEP protein of the A/equine/Nador/1/1997 (H3N8) strain against CRM1 has been studied by a molecular docking (protein-protein) method using Hex 8.0 software (Macindoe, Mavridis, Venkatraman, Devignes, \& Ritchie, 2010) without modifying the default settings.

\section{Molecular dynamics simulation}

The stability of the bonds in the model studied was made by molecular dynamics (MD) which could serve as a powerful tool for elucidating atomic biological behavior such as conformational changes and physiological repercussions (Klepeis, Lindorff-Larsen, Dror, \& Shaw, 2009).

The molecular dynamic simulation was performed by GROMOS using the server mmb.pcb.ub.es (Hospital et al., 2012). The simulation was used with an AMBER99SB force field and the following parameters: time 1000 (ps), $0.1 \Delta t$ (ps), output frequency 100 (steps), force constant $40\left(\mathrm{kcal} / \mathrm{mol} \times \AA^{2}\right)$, and distance between alpha carbon atoms 3.0 ( $\AA$ ). The mutations showed in alignment results were investigated in MD to study their effects on the structure of protein.

\section{Results}

Amplification and sequencing product

The partial sequence of the NS gene (segment 8) could be amplified for the three strains A/equine/Nador/1/ 1997 (accession no. JX182368.1 (693 bp)), A/equine/Essaouira/2/2004 (Q955611.1 (693 bp)), and A/equine/Essaouira/3/2004 (JQ955614.1 (693 bp)) (Boukharta et al., 2015). The sequence $693 \mathrm{bp}$ framed by the two primers included the sequence coding for the NS1 protein and partial NEP (NS2) (Figs. 1 and 2).

Partial NEP nucleotide sequences of the Moroccan equine influenza isolates (A/equine/Nador/1/1997, A/ equine/Essaouira/2/2004, and A/equine/Essaouira/3/ 2004) had been deduced, and their accession numbers are AFN69205, AFJ69908, and AFJ69912, respectively.

\section{Genomic and structural analysis}

Alignment result of the CRM1 domain amino acid sequences for the three strains of equine influenza isolated in Morocco showed that the two sequences of the CRM1-binding domain of the NEP strains of A/equine/ Essaouira/2/2004 (H3N8) and A/equine/Essaouira/3/ 2004 (H3N8) were identical and similar to the A/equine/ 

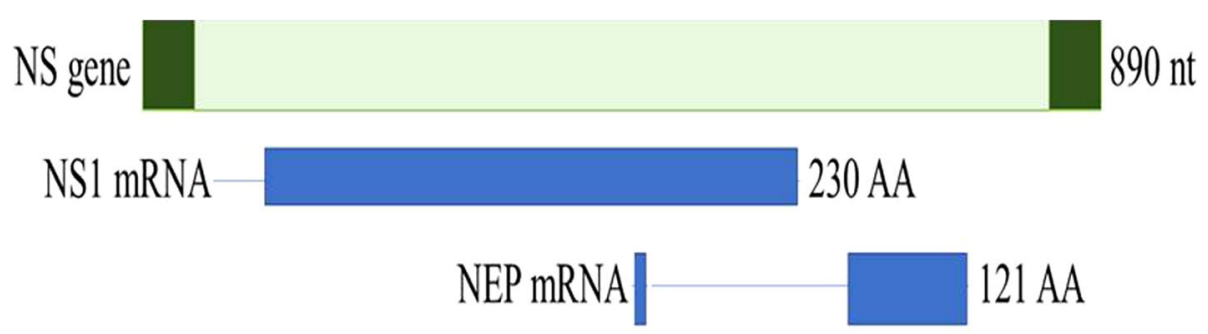

Fig. 1 Schematic representation of the non-structural protein of the influenza A virus

Miami/1/1963 (H3N8) reference strain. Due to these results, we studied the NEP of the reference strain. The partial NEP protein of A/equine/Nador/1/1997(H3N8) strain showed two mutations at the CRM1 domain at the $\mathrm{I} / 33 / \mathrm{T}$ and $\mathrm{R} / 34 / \mathrm{Q}$ residues (Fig. 3 ).

The results obtained from the BLAST (protein-protein) analysis and the homology revealed the non-existence of similar crystallized structures of the field studied in the available databases. However, for the equine CRM1 protein, it resembled the A unit of the crystallized protein 5 dis. 1 crystal structure of a human CRM1-RanGTP-SPN1 amino acid FG-repeat containing a fragment of human Nup214 (Fig. 4).

Due to the absence of homology that satisfied the abovementioned conditions of the NEP protein structures of strains A/equine/Nador/1/1997 (H3N8) and A/equine/ Miami/1/1963 (H3N8), the ab initio tools were used in order to predict and model the NEP structure using the I-TASSER server. The confidence in the quality of the predicted models provided a $C$-score equal to -1.40 for NEP A/equine/Miami/1/1963 (H3N8) and - 2.14 for NEP A/ equine/Nador/1/1997 (H3N8). According to Roy et al.,
2010, the $C$-score should be between -5 and 2 which means that these models are biologically significant (Roy et al., 2010). Then, for the topological similarity between the predicted model and the standard model expressed by the TM score, it is in order of $0.54 \pm 0.15$ and $0.5 \pm 0.15$ for NEP of strains A/equine/Miami/1/1963 (H3N8) and A/equine/Nador/1/1997 (H3N8), respectively. Similarly, the standard deviation parameter (RMSD) is about $7.3 \pm$ $4.2 \AA$ for A/equine/Nador/1/1997 (H3N8); however, it increases to $8.0 \pm 4.0 \AA$ in the NEP reference stain model, which increases the structural stability of the predicted model for the Nador strain (Fig. 5 and Table 1) and supports the stability of the protein structure in the biological system.

The model structures obtained were evaluated via Ramachandran and Progress server. The model NEP (A/equine/Miami/1/1963 (H3N8)) with an average quality of 2.5, a covalent binding quality of 3.5, and a non-covalent/packing of 1.5 was calculated by the PROSESS server tool. Identification of the residue is in the favored or unauthorized area of the Ramachandran plot (Figs. 2 and 3). These regions are defined

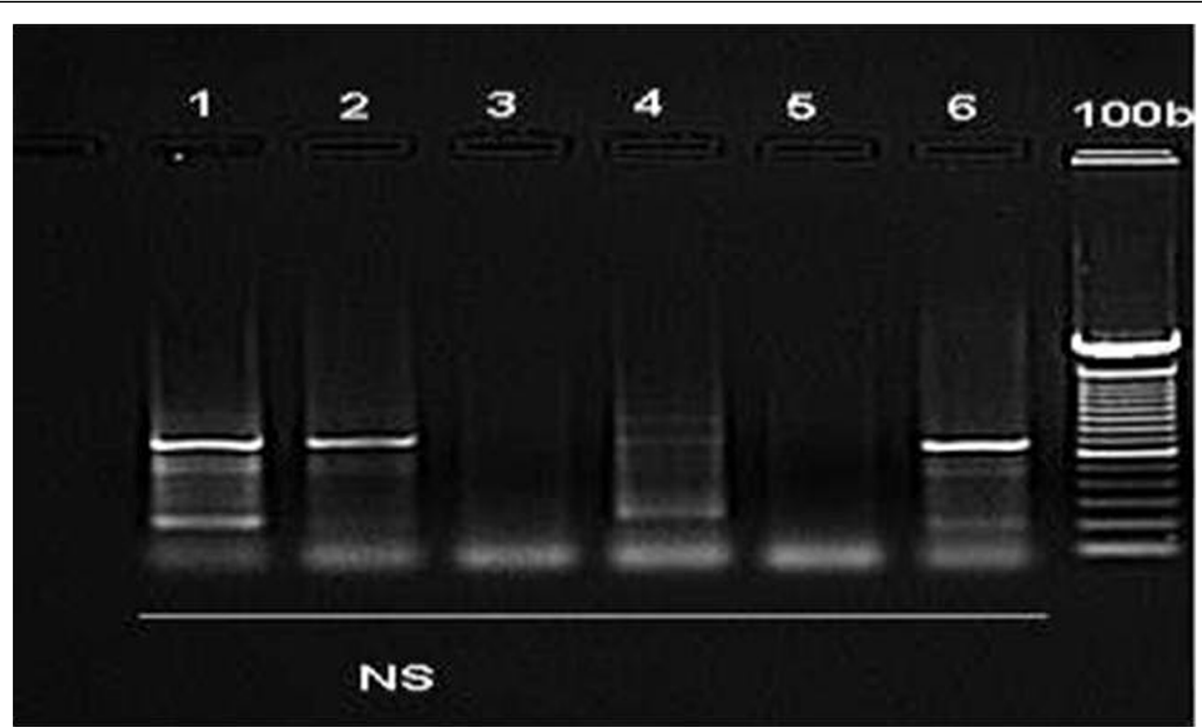

Fig. 2 1.5\% agarose gel electrophoresis of the RT-PCR amplification products for the gene encoding the NS protein from different samples. 1, A/ equine/Essaouira/2/2004; 2, Alequine/Nador/1/1997; 3-5, BEV isolates (bovine enteric virus) used as a negative control; 6, Alequine/Essaouira/3/2004 
Linking domain to CRM1

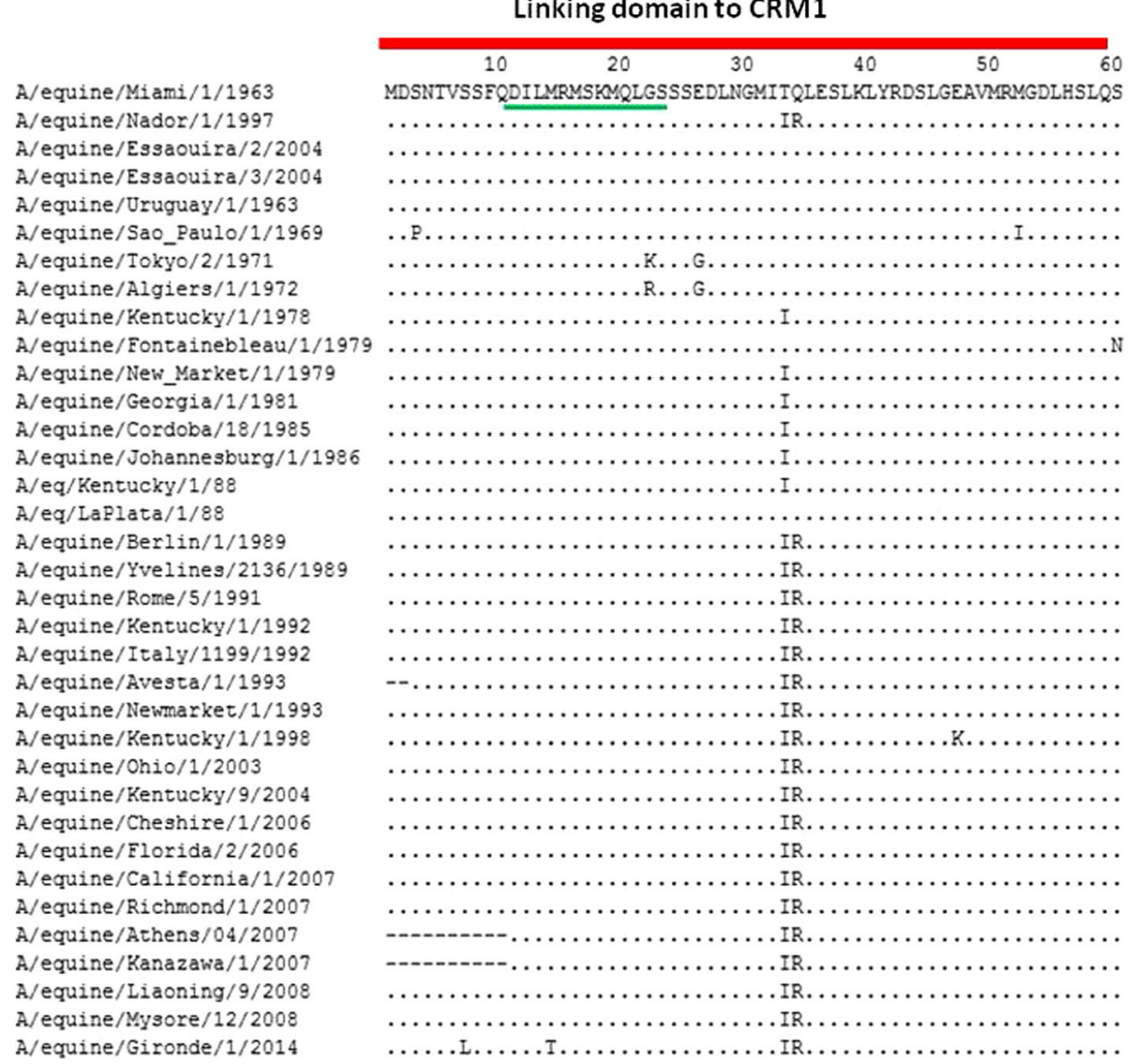

Fig. 3 Alignment of NEP sequences. NS2 protein (1-121) with reference strain A/ equine/Miami/1963 (the green line refers to the NES (nuclear export signal). NES, 11-23 aa; CRM1-binding domain, 1-58 aa; M1-binding domain, 59-116 aa (37). Accessions numbers: A/equine/Miami/1/1963, ABY81498; A/equine/Nador/1/1997, AFN69205; A/equine/Essaouira/2/2004, AFJ69908; A/equine/Essaouira/3/2004, AFJ69912; A/equine/Uruguay/1/ 1963, ACD85424; A/equine/Sao Paulo/1/1969, ACD85391; A/equine/Tokyo/2/1971, AEM60153; A/equine/Algiers/1/1972, ACF22132; A/equine/ Kentucky/1/1978, ABY81443; A/equine/Fontainebleau/1/1979, ACD85402; A/equine/New Market/1/1979, AEM60119; A/equine/Georgia/1/1981, ABY81531; A/equine/Cordoba/18/1985, ACD85281; A/equine/Johannesburg/1/1986, ACF22143; A/eq/Kentucky/1/88, AAC31253; A/eq/LaPlata/1/88, AAC31255; A/equine/Berlin/1/1989, ACD85413; A/equine/Yvelines/2136/1989, AAC31257; A/equine/Rome/5/1991, AAC31263; A/equine/Kentucky/ 1/1992, ACA24640; A/equine/Italy/1199/1992, ACD85314; A/equine/Avesta/1/1993, BAl67723;Alequine/Newmarket/1/1993, ACI48793;A/equine/ Kentucky/1/1998, ACH95656; A/equine/Ohio/1/2003, ABA42432; A/equine/Kentucky/9/2004, ACH95648;A/equine/Cheshire/1/2006, ACH95606; A/ equine/Florida/2/2006, ACH95626; Alequine/California/1/2007, ACH95622; Alequine/Richmond/1/2007, ACH95618; Alequine/Athens/04/2007, ADT80593; Avequine/Kanazawa/1/2007, BAF95466; Avequine/Liaoning/9/2008, ACE81911; Avequine/Mysore/12/2008, ACU82372; Avequine/Gironde/1/2014, APQ31497

using the ProCheck parameters (Morris, MacArthur, Hutchinson, \& Thornton, 1992).

The results showed that $88.5 \%$ of the residues were localized in the most favored regions and $11.5 \%$ of the residues in not favored regions of the Ramachandran plots (white area). The analysis of the index instability allowed the measurement of the protein stability in the test tube; this index is 53.79, which means that the NEPs have an average physiological stability and their predicted half-life is greater than $20 \mathrm{~h}$ in vivo which is more than $30 \mathrm{~h}$ in mammalian reticulocytes in vitro (Figs. 6 and 7).

Based on the results of the evaluation of the adopted models, these models can be reliably used if they meet an average structural quality.

\section{CRM1-NEP interaction}

To study the affinity between NEP and CRM1, we used docking to reveal the effects of mutations on the affinity between them. This was done by Hex 8.0 software with default parameters using 3D structure and fast Fourier transform (FFT) docking methods shape + electro + DARS.

The results of docking after seven replicates demonstrated that NEP (A/equine/Nador/1/1997 (H3N8) had been more refined to CRM1 with a total interaction energy of $-814.3 \mathrm{kcal} / \mathrm{mol}$. However, it is $3657.7 \mathrm{kcal} / \mathrm{mol}$ for NEP (A/equine/Miami/1/1963 (H3N8), and RMSD are less than $\AA$.

These data revealed that the nature of the reactions between the NEP and CMR1 proteins through the two 


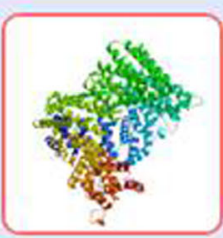

Model 01 B -
Oligo-State 0 Ligands

MONOMER 1 MMAL $^{\odot}, 1 \times \mathrm{PRO}^{\odot} \wedge$

$1 \times$ MALTOSE

Ligand 2 in contact with: Chain A: $\$ 490$, W491, K492

$1 \times$ PROLINE

Ligand 1 in contact with: Chain A : E428, N495, W499, S542,

N543, Y546

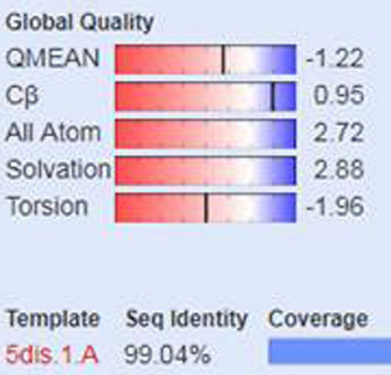

5dis. 1 .A $99.04 \%$
GMQE $\odot$ QMEAN $\theta$

0.98

$-1.221 B$

\section{(Moder}
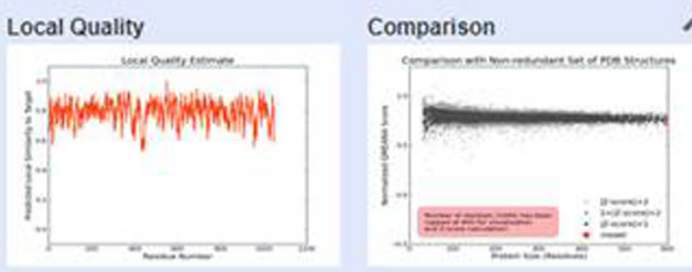

Description

Exportin-1

Fig. 4 Result of homology of the sequence CRM1 obtained by SWISS-MODEL. The figure demonstrates a great similarity between the equine CRM1 sequence and the crystallized structure 5 dis.1 in its unit A (structure CMR1 homology 5 dis.1. crystal structure of CRM1RanGTP-SPN1-repeat containing fragment of Nup214 to 99\%), and the local quality of the structure demonstrates a theoretical stability as well as statistical significance which is less than 0.0001 . This statistical significance allows reducing the random distribution of optimal global alignment scores (https://swissmodel.expasy.org/)

mutations passes from an exothermic reaction requiring energy to an endothermic regimen releasing energy for NEP (A/equine/Nador/1/1997(H3N8), which promotes energy interactions with NEP A/equine/Nador/1/1997 (H3N8) and NEP A/equine/Miami/1/1963 (H3N8) (Fig. 8).
This confirmed that the mutations developed in equine influenza viruses are adaptive mutations for the virus to its equine cellular environment, knowing that the A/equine/Miami/1/1963 (H3N8) strain is of avian origin.
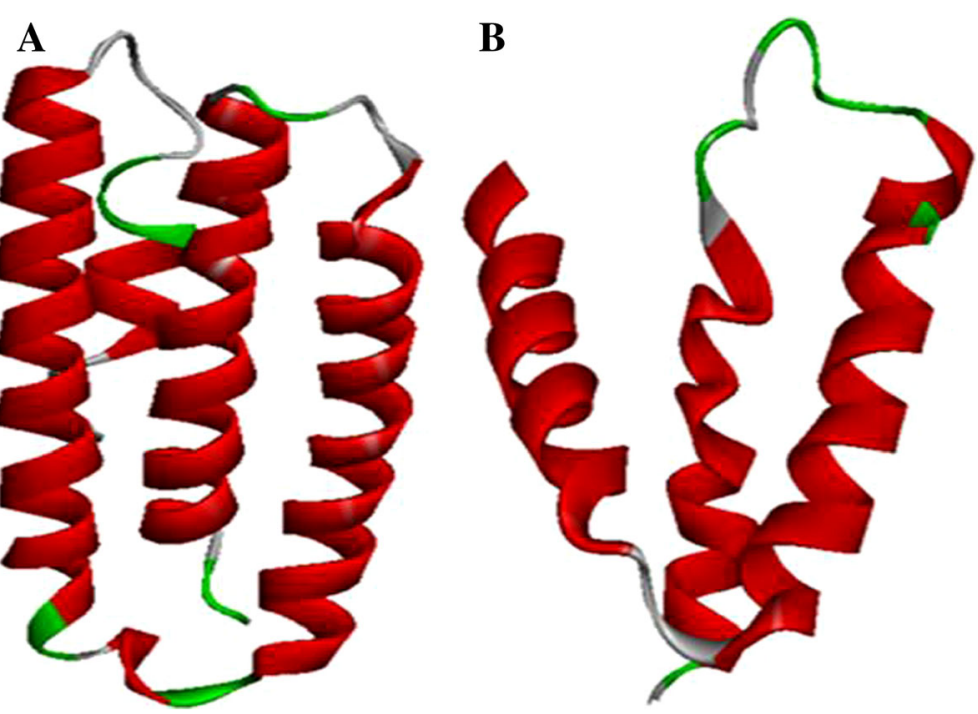

Fig. 5 Structure modeled by ab initio CRM1-binding domain of the NEP of both A/equine/Miami/1/1963 (H3N8) reference strain (a) and A/ equine/Nador/1/1997 (H3N8) (b). These two structural models well illustrate the effect of mutations resulting in a V-modification of the threedimensional structure of the CRM1 domain of NEP of A/equine/Nador/1/1997 strain (H3N8) 
Table 1 Result of docking protein-protein from Hex 8.0 software (http://hex.loria.fr/)

\begin{tabular}{|c|c|c|c|c|c|c|c|c|}
\hline C1st strains & Soln & Models & E. total & E. shape & E. force & E. air & Bmp & RMS \\
\hline A/equine/Miami/1/1963 & 1 & 000:000 & 3657.7 & 3657.7 & 0.0 & 0.0 & 97 & -1.00 \\
\hline A/equine/Nador/1/1997 & 1 & 1000:000 & -814.3 & -814.3 & 0.0 & 0.0 & -1 & -1.00 \\
\hline
\end{tabular}

Modeling results showed that the mutated sequences having the I/33/T and R/34/Q substitutions are mutations of adaptations favorable to the NEP-CRM1 interaction; according to the alignment figure, it is evident that the evolution of the mutations was initiated by the mutation I/ 33/T (1963-1988), and then, the mutation was reinforced by a second one $\mathrm{R} / 34 / \mathrm{Q}$; this suggests that the virus had gradually adapted to the new host species.

\section{Discussion}

In this context, our study aimed to demonstrate the impact of the genetic mutations of the NEP on the three-dimensional structure (3D) and contributed to explain how those mutations influenced the pathogenesis mechanism.

This study aimed to report the impact of mutations on the three-dimensional structure of CRM1 of NEP proteins
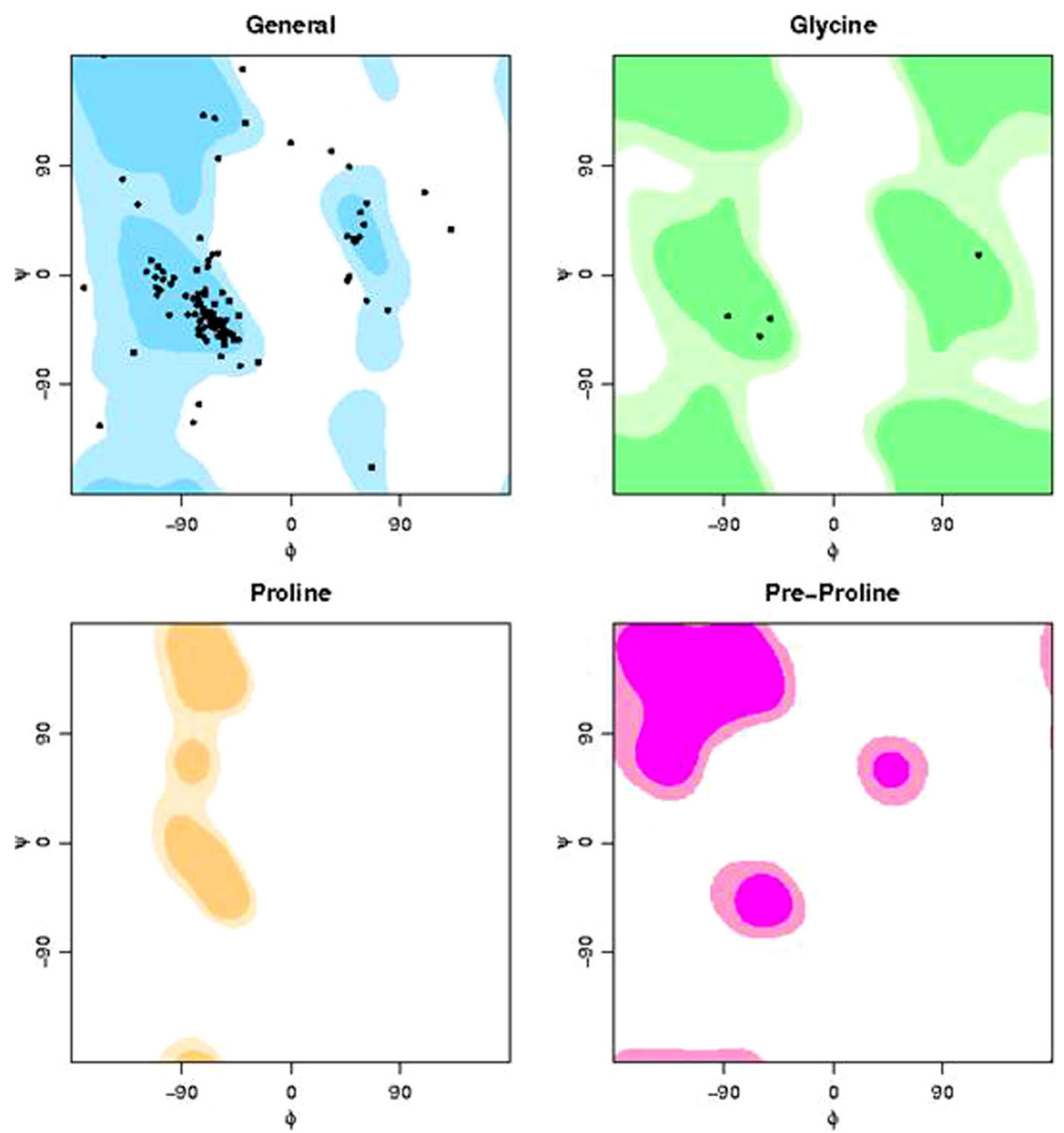

Fig. 6 Ramachandman plot of the reference sequence. The CRM1 domain of the A/equine/Miami/1/1963(H3N8) reference strain is characterized by good quality in the disposition of the residues. Most of the amino acids are located inside the intrigues $(0,-90)$ for the alpha helices and $(30,90)$ beta sheets. The graph (or diagram) of Ramachandran can visualize all the possible combinations of dihedral angles $\Psi$ (psi) against $\Phi$ (phi) in the amino acids of a polypeptide, and that contribute to the conformation of the structure of the proteins. This graph allows, therefore, to approximate a priori what will be the secondary structure of the peptide, since there are combinations of typical angles for each structure ( $a$-helix and $\beta$-sheet). The conformation of the peptides is defined by assigning values for each pair of corners $\Phi i, \psi_{i}$ for each amino acid. In the second quadrant are the combinations of the leaf $\beta$, in the third quadrant are the right a helix and the loops; in the first quadrant the combinations of the left a helix 

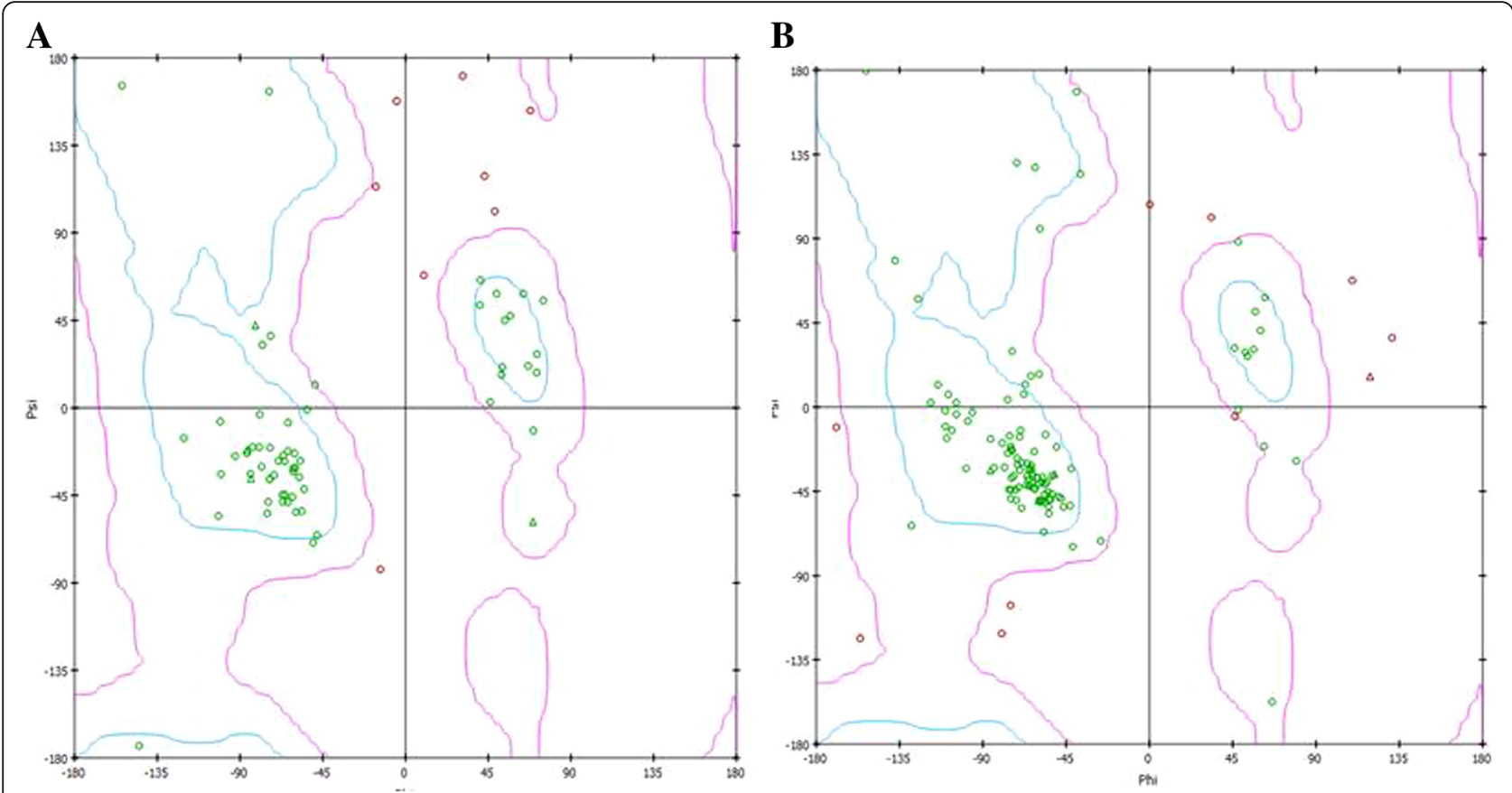

Fig. 7 Comparative Ramachandan plot of the two modeled domains by I-TASSER. a NEP protein of strain A/equine/Nador/1/1997(H3N8). b NEP protein of reference strain A/equine/Miami/1/1963 (H3N8): NEP structure of A/equine/Nador/1/1997 (H3N8). The results shown that NEP of Nador strain is characterized by a higher structural stability compared to that of Miami. This is explained by the ratio of the amino acids (black dot in two regions alpha and beta of protein). In Ramachandan plot was found that the upper left region could be divided into two: one on the left containing amino acids in beta leaves, and the other on the right with amino acids in random tangles

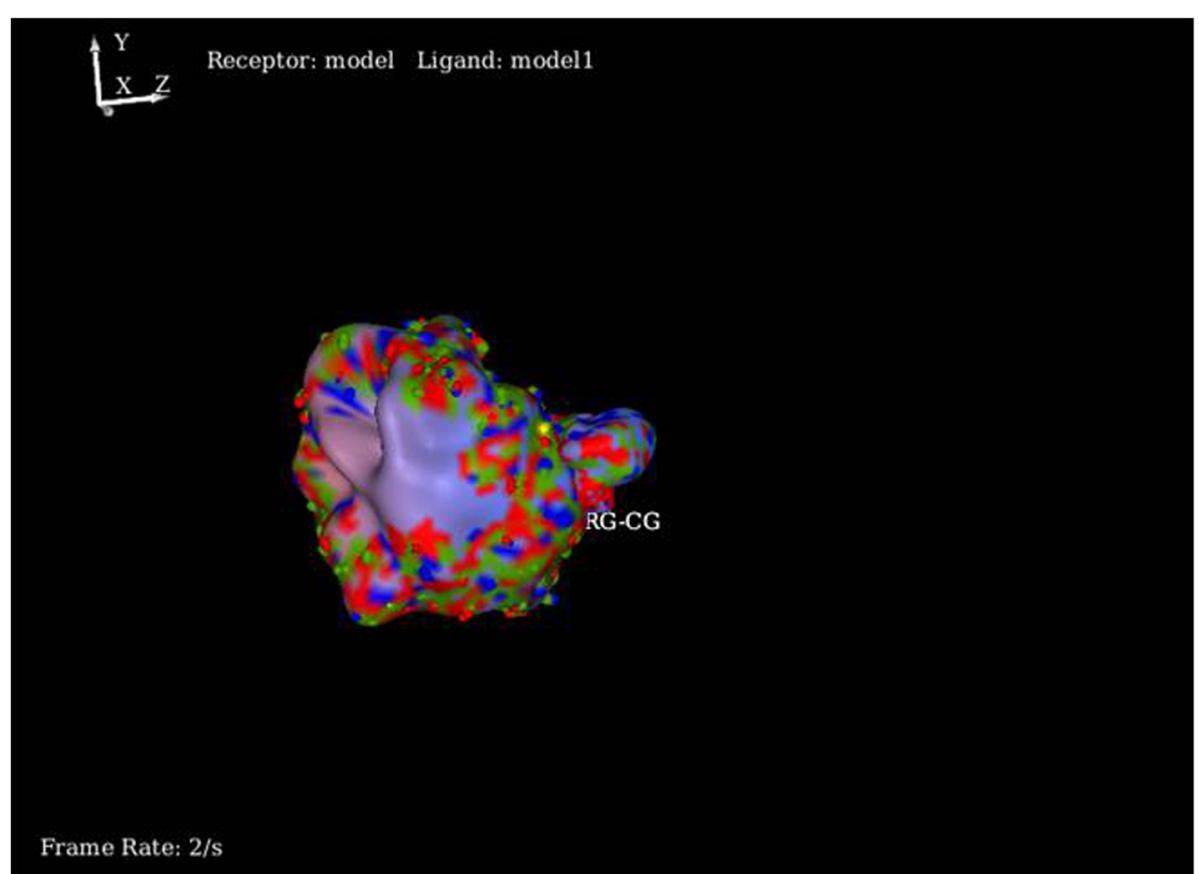

Fig. 8 Interaction between CRM1 (big macromolecule in the left) and NEP of Miami (small macromolecule in the right-RG-CG). The interaction between the two macromolecules demonstrates such a difference in the structural complementarity between them 
of three strains of equine influenza virus (H3N8) isolated in Morocco using computer tools and in silico. Compared to the reference strain, the two strains of Essaouira showed no mutations at the domain-binding CRM1 of the NEP protein, while the A/equine/Nador/1/1997 strain (H3N8) had two mutations at the $\mathrm{T} / 33 / \mathrm{I}$ and $\mathrm{Q} / 34 / \mathrm{R}$ residues.

Computational tools allowed the study of the effect of those mutations in e3D structure and evaluated computationally their pathogenesis mechanism before and after the mutation.

The alignment of 189 amino acid sequences of the NEP domain of available equine influenza viruses on the NCBI database (data not shown) revealed that these two mutations (I/33/T, R/34/Q) were frequently reported in the most equine influenza viruses isolated after 1989, time of onset divergence of the H3N8 virus in two distinct lineages of American and European. The NEP of the A/equine/Miami/1/1963 (H3N8) strain and those in the pre-divergence evolution phase did not simultaneously show two types of substitutions, sometimes the I/ $33 / \mathrm{T}$ mutation but very rarely the $\mathrm{R} / 34 / \mathrm{Q}$ mutation. After its first passage, the equine influenza virus originating from the avian pole took several years of evolution within the new host (Equidae) before the occurrence of these two mutations. Those mutations affected the protein in its three-dimensional structure and provided the virus the ability to adapt to its cellular environment and to evade the immune system as it is capable of altering its own protein.

These two mutations corresponded to an adaptation phenomenon of the equine cellular environment, since they support the interaction between the protein NEP and CRM1 with a greater affinity compared to the reference strain. The three-dimensional modification of the NEP protein of the A/equine/Nador/1/1997 strain further facilitates nucleocytoplasmic trafficking in equine species.

\section{Conclusion}

The mutations assigned to 33 and 34 residues are recognized as adaptation mutations, favoring the interaction between the viral vRNPs and the CRM1 and facilitating the nuclear export of the newly formed viral constituents, consequently the viral assembly in the cytoplasm. Globally, these two mutations are part of the adaptation of equine influenza's avian origin strains to equine species.

These reported mutations affect the virulence of the strains, which corroborates the exacerbation of virulence as well as the pathogenic effect detected in the field.

\section{Abbreviations}

CRM1: Chromosome maintenance protein 1; NEP: Nuclear export protein; NPC: Nuclear pore complex; RanGTP: RAs-related nuclear protein GTP; VRNP: Viral ribonucleoprotein complex

\section{Acknowledgements}

This study was supported by the Laboratory of Virology, Microbiology, Quality and Biotechnologies/Ecotoxicology and Biodiversity (School of Sciences and Techniques), University Hassan II of Casablanca, Ministry of Higher Education in Morocco, and Laboratory of Genetics of the Royal Gendarmerie of Morocco. The authors thank also the National Center for Scientific and Technical Research (CNRST-UATRS) from Morocco for their technical support.

\section{Funding}

This work did not receive any specific funding. However, the University Hassan II of Casablanca kindly supported the research at the Laboratory of Virology, Microbiology, Quality and Biotechnologies/Ecotoxicology and Biodiversity (Faculty of Sciences and Techniques) covering the services fees of the sequencing and phylogeny analysis performed at the National Center for Scientific and Technical Research (CNRST) Rabat. In addition, the financial and technical support of the viral isolation was realized with the support of BIOPHARMA Society. However, no institutional funding was available to cover publication costs.

\section{Availability of data and materials}

The data in this manuscript are available at the Laboratory of Virology, Microbiology, Quality and Biotechnologies/ Ecotoxicology and Biodiversity (Faculty of Sciences and Techniques, University Hassan II of Casablanca), BIOPHARMA Society, and the National Center for Scientific and Technical Research (CNRST) from Morocco.

\section{Authors' contributions}

The work presented here was carried out in collaboration between all authors. $M B$ is the principal investigator who performed and wrote the first draft of the manuscript. YK used the bioinformatics methods, FZ helped the redrafting of the manuscript, and HEA, MME conceived of the study, MME is the principal investigator as well, followed all the stages of writing and finalization of this manuscript, and helped the revision of the manuscript. All authors read and approved the final manuscript.

\section{Authors' information \\ N/A.}

\section{Ethics approval and consent to participate}

Samples from sick animals are sent to BIOPHARMA for diagnosis, and biosafety conditions have been respected in accordance with the rules required by the OIE Terrestrial Manual. (Chapter 1.1.4: Biosafety and biosecurity: Standard for managing biological risk in the veterinary laboratory and animal facilities (http://www.oie.int/en/international-standard-setting/ terrestrial-manual/access-online/), accessed 23 November 2017). Equine influenza virus was isolated from embryonated eggs and MDCK cells in containment level 3 (P3). The usefulness and design of the research were validated by the veterinary research team in virology of BIOPHARMA, respecting the ethical rules implemented.

\section{Consent for publication}

This manuscript does not contain individual data and case reports.

\section{Competing interests}

The authors declare that they have no competing interests.

\section{Publisher's Note}

Springer Nature remains neutral with regard to jurisdictional claims in published maps and institutional affiliations.

\section{Author details}

'Genetic Laboratory of Royal Gendarmerie, Rabat, Morocco. 'Laboratory of Virology, Microbiology, Quality and Biotechnologies/Ecotoxicology and Biodiversity-Team of Virology, Oncology and Medical Biotechnologies, Faculty of Sciences and Techniques, University Hassan II of Casablanca, P O BOX. 146, 20650 Mohammedia, Morocco. 
Received: 11 June 2018 Accepted: 3 April 2019

Published online: 29 April 2019

\section{References}

Akarsu, H., Burmeister, W. P., Petosa, C., Petit, I., Müller, C. W., Ruigrok, R. W., \& Baudin, F. (2003). Crystal structure of the M1 protein-binding domain of the influenza A virus nuclear export protein (NEP/NS2). The EMBO Journal, 22, 4646-4655

Berjanskii, M., Liang, Y., Zhou, J., Tang, P., Stothard, P., Zhou, Y., \& Wishart, D. S. (2010). PROSESS: a protein structure evaluation suite and server. Nucleic Acids Research., 38, 633-640.

Biasini, M., Bienert, S., Waterhouse, A., Arnold, K., Studer, G., Schmidt, T., .. Schwede, T. (2014). SWISS-MODEL: modelling protein tertiary and quaternary structure using evolutionary information. Nucleic Acids Research, 42, 252-258.

Boukharta, M., Azlmat, S., Elharrak, M., \& Ennaji, M. M. (2015). Multiple alignment comparison of the non-structural genes of three strains of equine influenza viruses (H3N8) isolated in Morocco. BMC Research Notes, 24, 471-478.

Boukharta, M., Touil, N., El Fahim, E., Terta, M., Kissi, B., Loutfi, C., ... Ennaji, M. M. (2013). Phylogenetic and molecular characterization of the equine influenza virus A (H3N8) causing the 1997 and 2004 outbreaks in Morocco. Journal of Equine Veterinary Science, 34, 942-948.

Brown, E. G. (2000). Influenza virus genetics. Biomedicine \& Pharmacotherapy, 54, 196-209.

Castrucci, M. R., \& Kawaoka, Y. (1995). Reverse genetics system for generation of an influenza A virus mutant containing a deletion of the carboxyl-terminal residue of M2 protein. Journal of Virology, 69, 2725-2728.

Chen, J., Skehel, J. J., \& Wiley, D. C. (1999). N- and C-terminal residues combine in the fusion-pH influenza hemagglutinin HA (2) subunit to form an $\mathrm{N}$ cap that terminates the triple-stranded coiled coil. Proceedings of the National Academy of Sciences of the United States of America, 96, 8967-8972.

Darapaneni, V., Prabhaker, V. K., \& Kukol, A. (2009). Large-scale analysis of influenza $A$ virus sequences reveals potential drug target sites of nonstructural proteins. Journal of General Virology, 90, 2124-2133.

Dea, S., Elazhary, M. A. S. Y., \& Roy, R. S. (1980). Les virus influenza chez 1'homme et les animaux Une revue de la littérature. The Canadian Veterinary Journal, 21, 171-178.

Elton, D., Medcalf, L., Bishop, K., Harrison, D., \& Digard, P. (1999). Identification of amino acid residues of influenza virus nucleoprotein essential for RNA binding. Journal of Virology, 73, 7357-7367.

Fornerod, M., Ohno, M., Yoshida, M., \& Mattaj, I. W. (1997). CRM1 is an export receptor for leucine-rich nuclear export signals. Cell, 90, 1051-1060.

Hospital, A., Andrio, P., Fenollosa, C., Cicin-Sain, D., Orozco, M., \& Gelpí, J. L. (2012). An integrated web-based platform for molecular dynamics simulations. Bioinformatics, 28, 1278-1279.

Ito, T., Kawaoka, Y., Ohira, M., Takakuwa, H., Yasuda, J., Kida, H., \& Otsuski, K. (1999). Replacement of internal protein genes, with the exception of the matrix, in equine 1 viruses by equine 2 influenza virus genes during evolution in nature. The Journal of Veterinary Medical Science, 61, 987-989.

Iwatsuki-Horimoto, K., Horimoto, T., Fujii, Y., \& Kawaoka. Y. (2004). Generation of Influenza A Virus NS2 (NEP) Mutants with an Altered Nuclear Export Signal Sequence. Journal of Virology, 78 (18), 10149-10155.

Kissi, B., Daoudi, N., El kantour, A., Id sisi yahia, K., \& Benazzou, H. (1998). First isolation in the Morocco of the equine influenza virus called $\mathrm{A} /$ Equi/ Morocco/1/97Esp. Veterinary, 14, 10.

Klaus, K., Ruigrok, R. W. H., \& Baudin, F. (1997). Roles of the influenza virus polymerase and nucleoprotein in forming a functional RNP structure. The EMBO Journal, 16, 1248-1257.

Klepeis, J. L., Lindorff-Larsen, K., Dror, R. O., \& Shaw, D. E. (2009). Long-timescale molecular dynamics simulations of protein structure and function. Current Opinion in Structural Biology, 19, 120-127.

Macindoe, G., Mavridis, L., Venkatraman, V., Devignes, M. D., \& Ritchie, D. W. (2010). HexServer: an FFT-based protein docking server powered by graphics processors. Nucleic Acids Research, 38, 445-449.

Mena, I., Jambrina, E., Albo, B., Perales, C., Ortin, J., Arrese, M., ... Portela, A. (1999). Mutational analysis of influenza A virus nucleoprotein: identification of mutations that affect RNA replication. Journal of Virology, 73, 1186-1194.

Morris, A. L., MacArthur, M. W., Hutchinson, E. G., \& Thornton, J. M. (1992). Stereochemical quality of protein structure coordinates. Proteins, 12, 345-364.

Myers, C., \& Wilson, D. (2006). Equine Influenza Virus. Clinical Techniques in Equine Practice, 5, 187-196.
Neumann, G., Brownlee, G. G., Fodor, E., \& Kawaoka, Y. (2004). Orthomyxovirus replication, transcription, and polyadenylation. Current Topics in Microbiology and Immunology, 283, 121-143.

Neumann, G., Hughes, M. T., \& Kawaoka, Y. (2000). Influenza A virus NS2 protein mediates VRNP nuclear export through NES-independent interaction with hCRM1. The EMBO Journal, 19, 6751-6758.

Rott, R., Orlich, M., \& Scholtissek, C. (1979). Correlation of pathogenicity and gene constellation of influenza A viruses. III. Non-pathogenic recombinants derived from highly pathogenic parent strains. The Journal of General Virology, 44, $471-477$.

Roy, S., et al. (2010). Identification of functional elements and regulatory circuits by drosophila modencode. Science, 330, 1787-1797.

Skehel, J. J., \& Waterfield, M. D. (1975). Studies on the primary structure of the influenza virus hemagglutinin. Proceedings of the National Academy of Sciences, 72, 93-97.

Smirnova, Y. A., Fedorova, N. V., Ksenofontov, A. L., Kordyukova, L. V., Serebryakova, M. V., Baratova, L. A., \& Vaskovsky, B. V. (2009). Isolation of the influenza A HA2 C-terminal segment by combination of nonionic detergents. Advances in Experimental Medicine and Biology, 611, 311-312.

Sovinova, O., Tumova, B., Pouska, F., \& Nemec, J. (1958). Isolation of a virus causing respiratory disease in horses. Acta Virologica, 2, 52-61.

Temoltzin-Palacios, F. \& Thomas, D. B. (1994). Modulation of immunodominant sites in influenza hemagglutinin compromise antigenic variation and select receptor-binding variant viruses. Journal of Experimental Medicine, 179, 1719-1724.

Tissier, O. (2008). Comparative study of sequences of the equine influenza virus isolated in French. Thesis submitted to the University Claude Bernard - Lyon (medicine - pharmacy). http://www2.vetagro-sup.fr/bib/fondoc/th_sout/ phpdrupal.php?annee=2008.

Voeten, J. T. M., Bestebroer, T. M., Nieuwkoop, N. J. R., Fouchier, A. M., Osterhaus, A. D. M. E., \& Rimmelzwaan, G. F. (2000). Antigenic drift in the influenza A virus $(\mathrm{H} 3 \mathrm{~N} 2)$ nucleoprotein and escape from recognition by cytotoxic $T$ lymphocytes. Journal of Virology, 74, 6800-6807.

Waddell, G. H., Teigland, M. B., \& Sigel, M. M. (1963). A new influenza virus associated with equine respiratory disease. Journal of the American Veterinary Medical Association, 143, 587-590.

Watanabe, K., Takizawa, N., Katoh, M., Hoshida, K., Kobayashi, N., \& Nagata, K. (2001). Inhibition of nuclear export of ribonucleoprotein complexes of influenza virus by leptomycin B. Virus Research, 77, 31-42.

Webster, R. G. (1998). Influenza: an emerging disease. Emerging Infectious Diseases, $4,436-441$.

Zhang, Y. (2008). I-TASSER server for protein 3D structure prediction. BMC Bioinformatics, 9, 40.

\section{Submit your manuscript to a SpringerOpen ${ }^{\odot}$ journal and benefit from:}

- Convenient online submission

- Rigorous peer review

- Open access: articles freely available online

- High visibility within the field

- Retaining the copyright to your article

Submit your next manuscript at $>$ springeropen.com 